\title{
Isorhamnetin inhibits cell proliferation and induces apoptosis in breast cancer via Akt and mitogen-activated protein kinase kinase signaling pathways
}

\author{
SHAN HU, LIMING HUANG, LIWEI MENG, HE SUN, WEI ZHANG and YINGCHUN XU \\ Department of Breast and Thyroid Surgery, Shaoxing People's Hospital, \\ Shaoxing Hospital of Zhejiang University, Shaoxing, Zhejiang 312000, P.R. China
}

Received November 12, 2014; Accepted August 4, 2015

DOI: $10.3892 / \mathrm{mmr} .2015 .4269$

\begin{abstract}
Breast cancer is the most common cause of female cancer-associated mortality. Although treatment options, including chemotherapy, radiotherapy and surgery have led to a decline in the mortality rates associated with breast cancer, drug resistance remains one of the predominant causes for poor prognosis and high recurrence rates. The present study investigated the potential effects of the natural product, isorhamnetin on breast cancer, and examined the effects of isorhamnetin on the Akt/mammalian target of rapamycin (mTOR) and the mitogen-activated protein kinase (MAPK)/MAPK kinase (MEK) signaling cascades, which are two important signaling pathways for endocrine therapy resistance in breast cancer. The results of the present study indicate that isorhamnetin inhibits cell proliferation and induces cell apoptosis. In addition, isorhamnetin was observed to inhibit the Akt/mTOR and the MEK/extracellular signal-regulated kinase phosphorylation cascades. The inhibition of these two signaling pathways was attenuated by the two Akt and MEK1 inhibitors, but not by the nuclear factor- $\kappa \mathrm{B}$ inhibitor. Furthermore, epidermal growth factor inhibited the effects of isorhamnetin via activation of the Akt and MEK signaling pathways. These results indicate that isorhamnetin exhibits antitumor effects in breast cancer, which are mediated by the Akt and MEK signaling pathways.
\end{abstract}

\section{Introduction}

Breast cancer is the most common type of malignant tumor in females (1-3). Despite major advances in breast cancer screening, early diagnosis and treatment modalities, it remains the predominant cause of cancer-associated

Correspondence to: Dr Shan Hu, Department of Breast and Thyroid Surgery, Shaoxing People's Hospital, Shaoxing Hospital of Zhejiang University, 568 North Zhongxing Road, Shaoxing, Zhejiang 312000, P.R. China

E-mail: sxrmyyhs@163.com

Key words: isorhamnetin, breast cancer, Akt, mitogen-activated protein kinase kinase, apoptosis mortality in females worldwide (4). Existing treatment strategies include surgery, chemotherapy, endocrine therapy, and molecular-targeted therapy, which are limited by tumor recurrence and drug resistance (5-7). Therefore, novel approaches to enhance the effects of therapeutic agents and improve the existing standards of care are urgently required.

It is well-known that the phosphoinositide 3-kinase (PI3K)/Akt/mammalian target of rapamycin (mTOR) and the mitogen-activated protein kinase (MAPK)/MAPK kinase (MEK) signaling pathways have important roles in tumor progression and drug resistance in various types of cancer (4). Previous studies indicate that activation of the $\mathrm{PI} 3 \mathrm{~K} / \mathrm{Akt} / \mathrm{mTOR}$ signaling pathway is closely associated with the poor outcome of patients with breast cancer undergoing endocrine therapy $(8,9)$. The activation of this signaling pathway had been identified as an important mechanism of tamoxifen resistance (10-13). The MEK/MAPK signaling pathway has also been associated with tamoxifen resistance and chemoresistance $(14,15)$. In addition, the activation of the MEK/MAPK signaling pathway is involved in resistance to epidermal growth factor receptor (EGFR) tyrosine kinase inhibitor, gefitinib, in breast cancer cells (16). Therefore, compounds targeting these signaling pathways are likely to be promising agents against endocrine therapy resistance in breast cancer.

Natural products are widely administered to prevent cancer in multi-stage carcinogenesis in humans, and have been the subject of intensive research in recent years (17). Isorhamnetin is a flavonoid that is abundantly present in fruits, vegetables and tea, as well as in herbs that are used as traditional medicine, such as Ginkgo biloba extract and Persicaria thunbergii $\mathrm{H}(18,19)$. These two herbs are administered for the treatment of rheumatism, hemorrhage and cancer in traditional medicine (20-22). Isorhamnetin is the active compound of these herbal medicinal plants, and is an immediate metabolite of quercetin, also termed 3'-O-methylquercetin, which has been shown to inhibit various types of cancer, including esophageal (23) and gastric cancer (24), leukemia $(25,26)$, skin (27), colon (28) and lung cancer (29). However, to the best of our knowledge, no study to date has focused on the inhibitory effects of isorhamnetin on breast cancer, and the molecular mechanisms underlying its effects remain unclear. 
To better understand the mechanism underlying the effects of isorhamnetin on breast cancer, the present study examined the inhibition of isorhamnetin and the proliferation of various breast cancer cell lines, and explored the cell signaling pathways involved in its pharmacological effects.

\section{Materials and methods}

Cell lines. MCF7, T47D, BT474, BT-549, MDA-MB-231 and MDA-MB-468 breast cancer cell lines, as well as a MCF10A normal breast epithelial cell line (control) were purchased from the American Type Culture Collection (Manassas, VA, USA). The cells were routinely cultured in Dulbecco's modified Eagle's medium (DMEM) supplemented with $10 \%$ fetal bovine serum (Invitrogen Life Technologies, Carlsbad, CA, USA) at $37^{\circ} \mathrm{C}$ in a $5 \% \mathrm{CO}_{2}$ incubator. MCF7, T47D and BT474 are estrogen receptor (ER) and progestogen receptor (PR)-positive cells, and human epidermal growth factor (HER)2-negative cells. BT-549, MDA-MB-231 and MDA-MB-468 are ER-, PRand HER2-positive cells.

Reagents. Isorhamnetin was purchased from Shanghai Tongtian Biotechnology Co., Ltd. (Shanghai, China). Perifosine, PD184352 and JSH-23 were purchased from Selleck Chemicals (Houston, TX, USA). EGF was purchased from Sigma-Aldrich (St. Louis, MO, USA). The antibodies for mouse monoclonal $\beta$-actin (cat. no. 3700; $1: 1,000$ ), rabbit polyclonal phosphorylated (p)-EGFR immunoglobulin (Ig)G (cat. no. 2234; 1:1,000), rabbit monoclonal EGFR (cat. no. 4405; 1:1,000), rabbit monoclonal PI3K (cat. no. 4249; 1:1,000), rabbit monoclonal p-Akt (S473; cat. no. 4060; 1:500), mouse monoclonal Akt (cat. no. 2920; 1:1,000), mouse monoclonal p-ERK1/2 (cat. no. 9106; 1:500), rabbit monoclonal ERK1/2 (cat. no. 4695; 1:1,000) and rabbit monoclonal cleaved caspase-3 (cat. no. 9664; 1:500) were purchased from Cell Signaling Technology, Inc. (Danvers, MA, USA), and antibodies for rabbit monoclonal B cell lymphoma 2 (Bcl-2; cat. no. ab117115; 1:1,000), rabbit monoclonal Bcl-2-associated X protein (Bax; cat. no. ab32503; 1:1,000), rabbit monoclonal Bcl-extra large (xL; cat. no. ab32370; 1:2,000), rabbit monoclonal I $\kappa$ B (cat. no. ab32518; 1:1,000), rabbit monoclonal anti-NF-кB P65 antibody (cat. no. ab32536; 1:2,000) and rabbit polyclonal H3 (cat. no. ab1791; 1:2,000) were purchased from Abcam (Cambridge, MA, USA).

Cell Counting kit-8 (CCK-8) assay. The cells were seeded into 96 -well plates at a density of $5 \times 10^{3}$ cells/well in $100 \mu \mathrm{l}$ DMEM and placed in cell incubator for $12 \mathrm{~h}$ at $37^{\circ} \mathrm{C}$ in an atmosphere containing $5 \% \mathrm{CO}_{2}$. The cells were then treated with various concentrations of isorhamnetin (100, 33.3, 11.1, 3.7, $1.2,0.4$ and $0 \mu \mathrm{M}$ ) for $48 \mathrm{~h}$, and cell proliferation rates were determined by adding $10 \mu \mathrm{l}$ CCK- 8 solution (Dojindo Molecular Technologies, Inc., Kumamoto, Japan) prior to incubation at $37^{\circ} \mathrm{C}$ for $2 \mathrm{~h}$. The absorbance was measured at a wavelength of $450 \mathrm{~nm}$ using a SpectraMax 190 Microplate Reader (Molecular Devices, LLC, Sunnyvale, CA, USA). For each assay, four parallel wells were included, and the half maximal inhibitory concentration $\left(\mathrm{IC}_{50}\right)$ was measured using the inhibition curve and presented as the mean of three independent experiments.
A

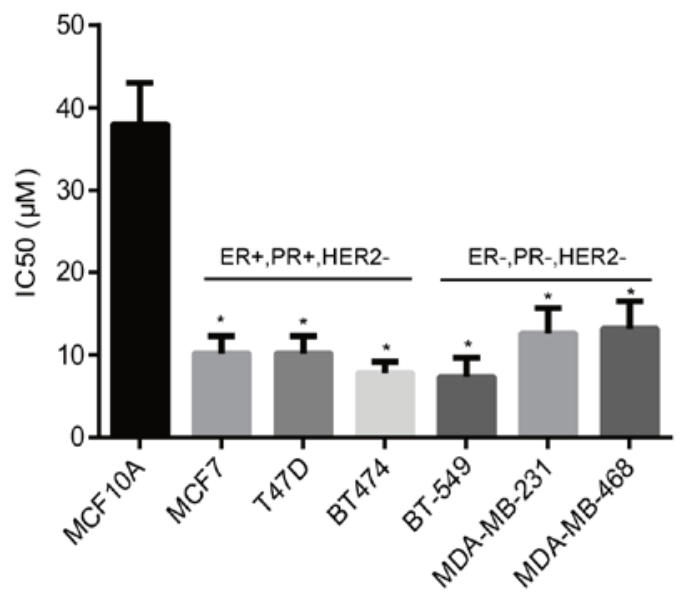

B

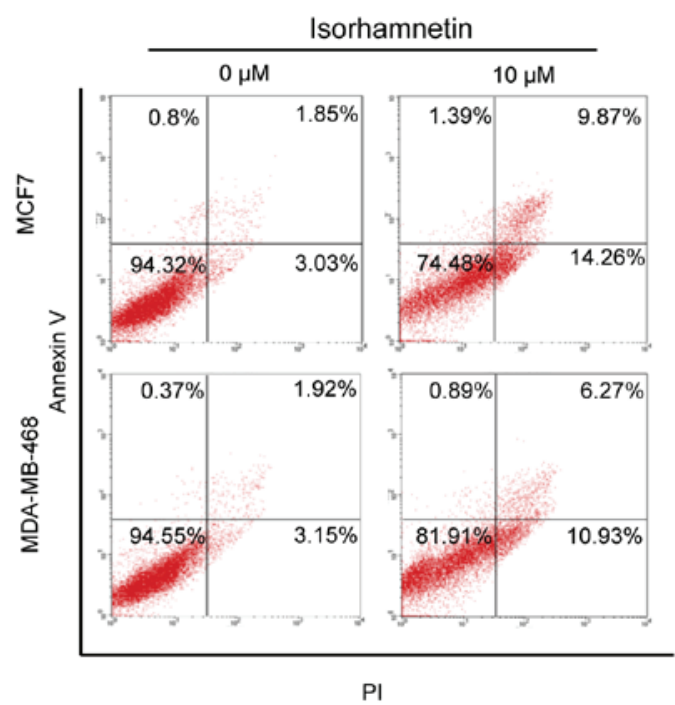

Figure 1. Isorhamnetin inhibits proliferation and induces apoptosis of breast cancer cells. (A) The cells were treated with various concentrations of isorhamnetin for $72 \mathrm{~h}$, cell proliferation was determined using a Cell Counting kit-8 (CCK-8) assay and the $\mathrm{IC}_{50}$ was calculated. ${ }^{*} \mathrm{P}<0.05, \mathrm{IC}_{50}$ of isorhamnetin in various cancer cells as compared with the MCF10A cells. (B) Isorhamnetin induced apoptosis of the MCF7 and MDA-MB-468 cells following a 48-h treatment. The upper right quandrant shows early apoptotic cells, the lower right the late apoptotic cells, the upper left quadrant indicates cell debris and the lower left the viable cells. $\mathrm{IC}_{50}$, half maximal inhibitory concentration; PI, propidium iodide.

Western blot analysis. The cells were washed twice with ice-cold phosphate-buffered saline (PBS) and harvested using radioimmunoprecipitation (RIPA) buffer (Beyotime Institute of Biotechnology; $50 \mathrm{mM}$ Tris, $150 \mathrm{mM} \mathrm{NaCl}$, $1 \%$ Triton $\mathrm{X}-100,0.1 \%$ SDS, and $1 \%$ sodium deoxycholate, with protease and phosphatase inhibitors). The cell lysate was placed in RIPA for $30 \mathrm{~min}$ and centrifuged at a speed of $12,000 \mathrm{x} \mathrm{g}$ for $15 \mathrm{~min}$, and the supernatant was then collected. Each aliquot of protein $(10 \mu \mathrm{g})$ was separated by $12 \%$ SDS-PAGE and transferred to Hybond-C nitrocellulose membranes (GE Healthcare Biosciences, Pittsburg, PA, USA) in transfer buffer (192 mM glycine, $25 \mathrm{mM}$ Tris, $2.5 \mathrm{mM}$ SDS, and 10\% methanol) (Sangon Biotech Co., Ltd., Shanghai, China). The membranes were blocked with $5 \%$ non-fat milk in Tris-buffered saline with 1\% Tween-20 (TBST; Sangon 


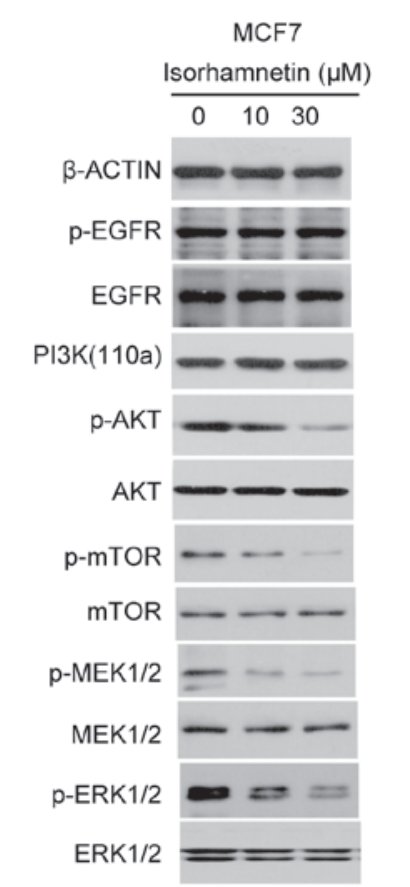

B

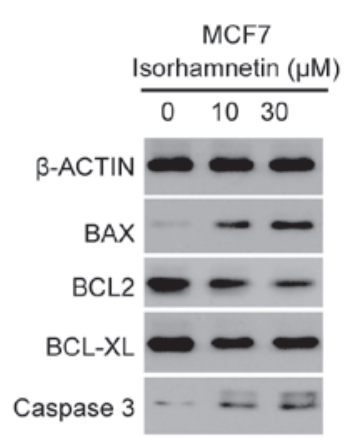

C

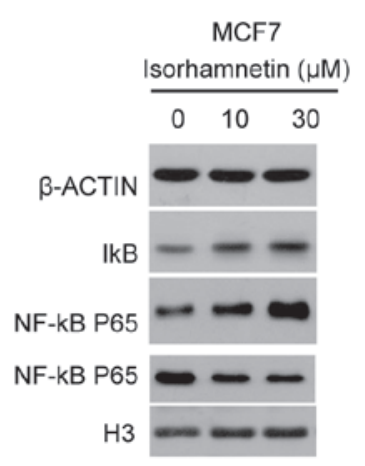

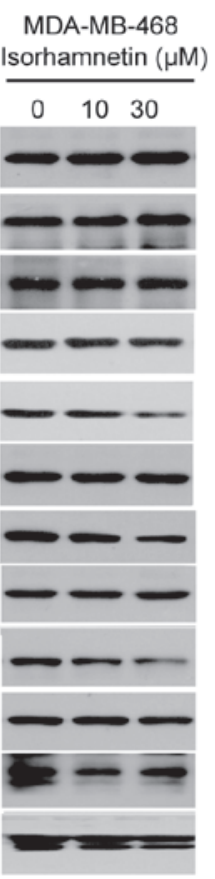
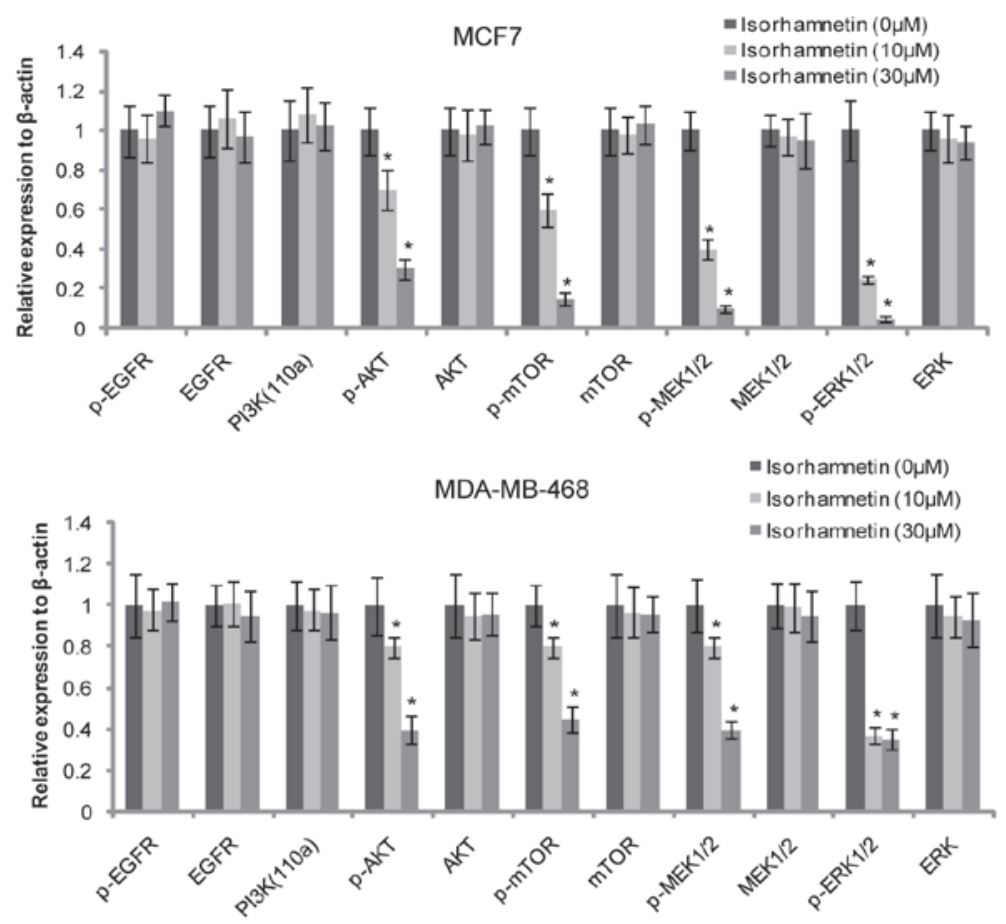

MDA-MB-468 Isorhamnetin $(\mu \mathrm{M})$
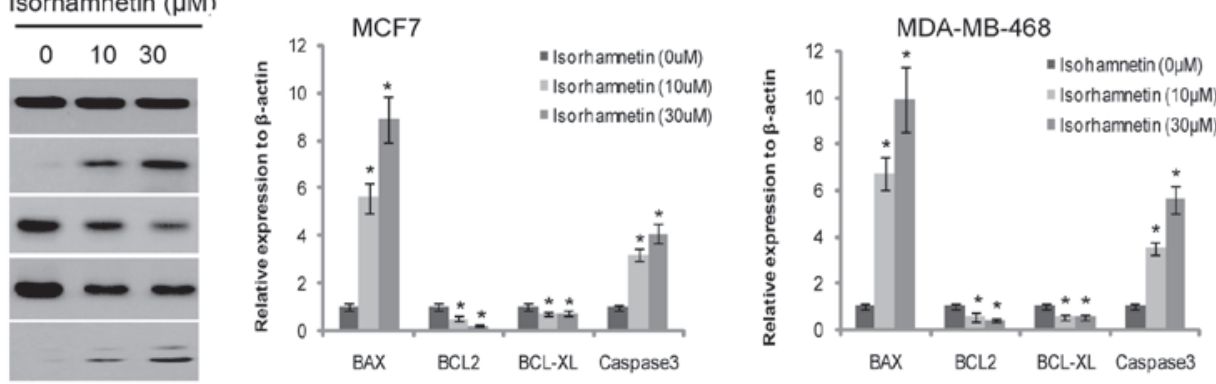

MDA-MB-468

Isorhamnetin $(\mu \mathrm{M})$
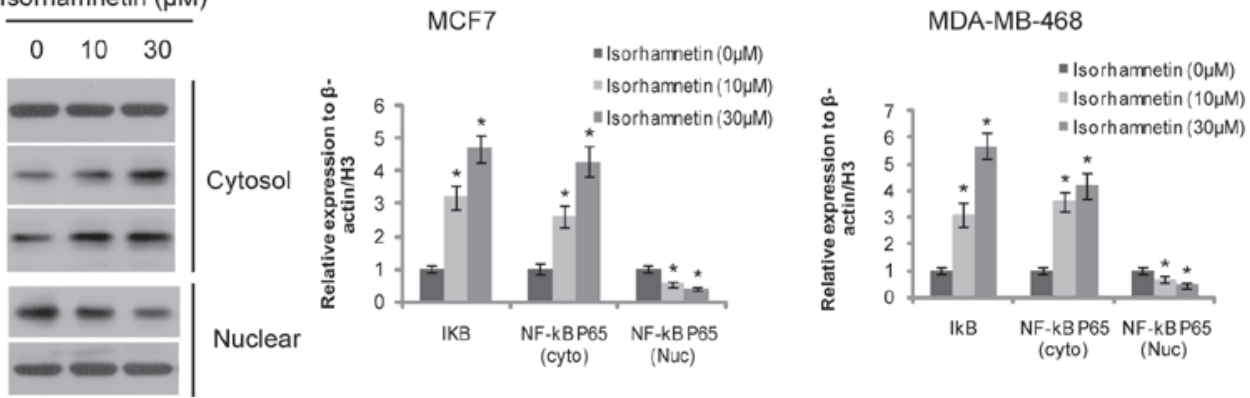

Figure 2. Effects of isorhamnetin on the cell signaling cascade. (A) Isorhamnetin inhibited the phosphorylation of Akt, mTOR, MEK1/2 and ERK1/2, but not of EGFR (12-h treatment). The protein expression levels were quantified by Image J, and presented as the relative expression levels to the control. (B) Isorhamnetin increased the expression level of Bax and cleaved caspase 3, and decreased the expression level of Bcl-2 and Bcl-xL (12-h treatment); (C) Isorhamnetin decreased the nuclear translocation of NF-kB (24-h treatment). p, phosphorylated; mTOR, mammalian target of rapamycin; MEK, mitogen-activated protein kinase kinase; ERK, extracellular signal-regulated kinase; EGFR, epidermal growth factor receptor; PI3K, phosphoinositide 3-kinase; Bcl-2, B cell lymphoma

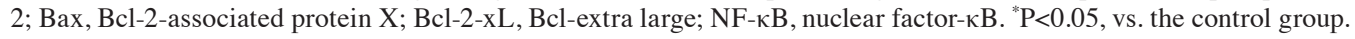

Biotech Co., Ltd.) for $1 \mathrm{~h}$ at room temperature, incubated with the previously mentioned primary antibodies at $4^{\circ} \mathrm{C}$ overnight, then washed three times with TBST for $10 \mathrm{~min}$ prior to incubation with secondary horseradish peroxidase-conjugated anti-rabbit (cat. no. 7074; Cell Signaling Technology, Inc.) or anti-mouse IgG (cat. no. 7076; Cell Signaling Technology,
Inc.) antibodies, and again washed three time with TBST for $10 \mathrm{~min}$. The blots were developed with an Enhanced Chemiluminescence Plus Western Blotting Detection system (GE Healthcare Biosciences). The total protein was determined using the Bicinchoninic Acid method (Invitrogen Life Technologies; cat. no. 23235). 
A
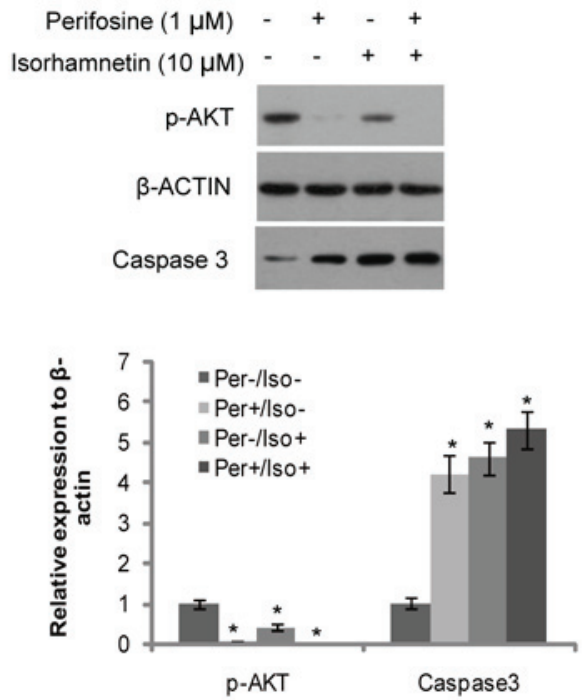

C

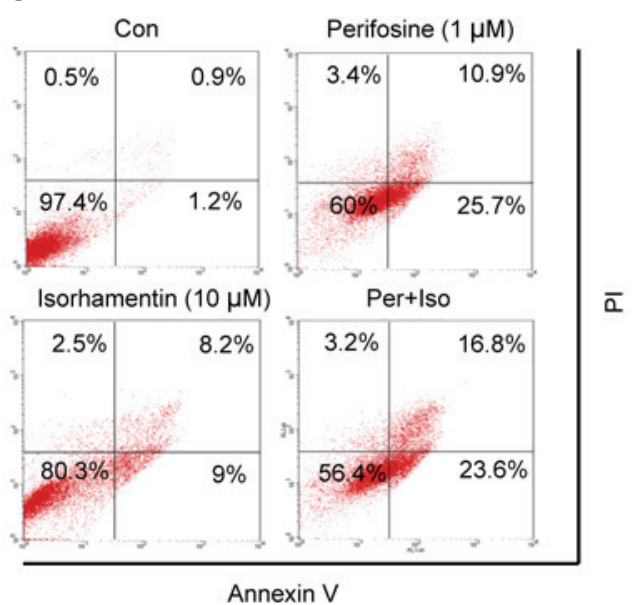

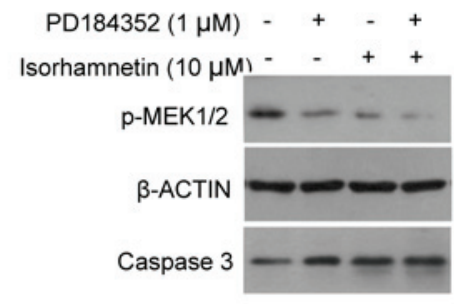

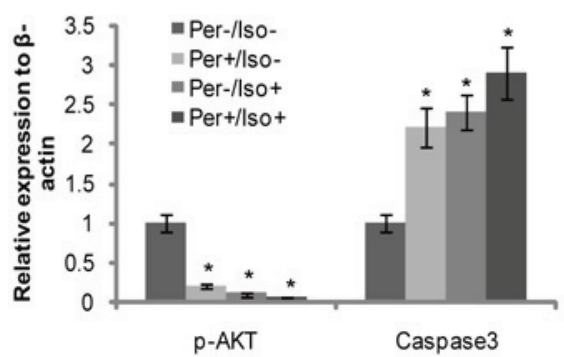

D

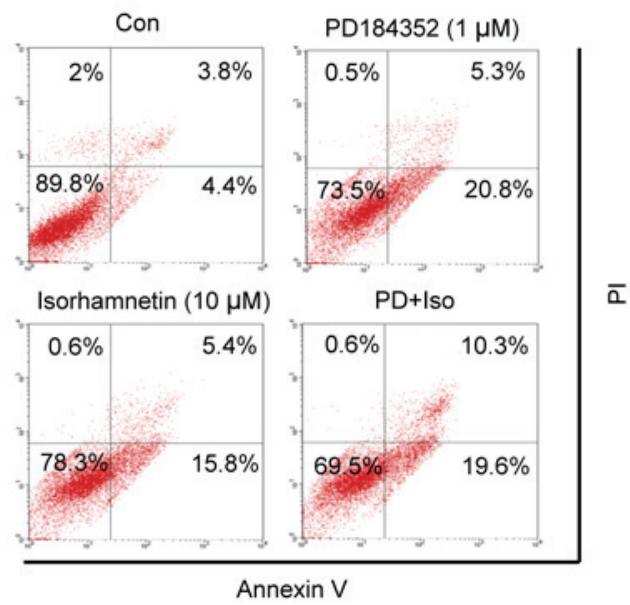

Figure 3. Induction of apoptosis was attenuated by the Akt and MEK inhibitors in the MCF7 cells. (A) Induction of caspase-3 cleavage was attenuated by the Akt inhibitor, perifosine (12-h treatment). The protein expression levels were quantified by Image $\mathrm{J}$, and presented as the relative expression levels to the control. (B) Induction of caspase-3 cleavage was attenuated by the MEK inhibitor, PD184352 (12-h treatment). "P $<0.05$, vs. the control group. (C) Induction of cell apoptosis was attenuated by the Akt inhibitor, perifosine (48-h treatment). (D) Induction of cell apoptosis was attenuated by the MEK inhibitor, PD184352 (48-h treatment). MEK, mitogen-activated protein kinase kinase; Con, control; PI, propidium iodide; PD, PD184352; Per, perifosine; Iso, isorhamnetin. The upper right quadrant shows the early apoptotic cells, the lower right the late apoptotic cells, the upper left quadrant indicates cell debris and the lower left the viable cells.

Flow cytometric assay. To determine the effects of isorhamnetin on cell apoptosis, the MCF7 or MDA-MB-468 cells were seeded at a density of $2 \times 10^{5}$ cells/well in a 6 -well plate, and incubated at $37^{\circ} \mathrm{C}$ overnight. The cells were treated with isorhamnetin or the inhibitors (perifosine, PD184352 and JSH-23) for $48 \mathrm{~h}$, prior to being detached and washed with cooled PBS. The cells were collected by trypsin (Invitrogen Life Technologies) digestion and centrifugation at $300 \mathrm{x} \mathrm{g}$ for $3 \mathrm{~min}$. The cells were then resuspended in binding buffer containing Annexin-V and propidium iodide (PI; Beyotime Institute of Biotechnology, Jiangsu, China) and incubated for $15 \mathrm{~min}$ in the dark at room temperature. Analysis was performed using a FACSCalibur analyzer (BD Biosciences, San Jose, CA, USA).

Statistical analysis. Protein expression was quantified using Image J software (version 1.31; Utrecht University, Utrecht,
Netherlands) and expressed as the relative expression levels to the control group. P-values were calculated by comparison to the control group using analysis of variance with SPSS 19 (IBM SPSS, Armonk, NY, USA). P<0.05 was considered to indicate a statistically significant result.

\section{Results}

Isorhamnetin inhibits proliferation and induces apoptosis of breast cancer cells. The inhibitory effects of isorhamnetin on breast cancer cells were determined using the CCK-8 method. As shown in Fig. 1A, isorhamnetin inhibited the proliferation of numerous breast cancer cells $\left(\mathrm{IC}_{50}, \sim 10 \mu \mathrm{M}\right)$, including MCF7, T47D, BT474, BT-549, MDA-MB-231 and MDA-MB-468, whereas less inhibitory activity was observed in the MCF10A normal breast epithelial cell line $\left(\mathrm{IC}_{50}, 38 \mu \mathrm{M}\right)$. These results indicated that isorhamnetin induces pronounced 
inhibitory effects on breast cancer cell lines $(\mathrm{P}<0.05)$, as compared to normal breast epithelial cell lines, which suggests that isorhamnetin may act on the activation pathway of cancer cells. The effect of isorhamnetin on cell apoptosis was subsequently determined using two breast cancer cell lines, MCF7 and MDA-MB-468. Isorhamnetin markedly promoted cell apoptosis of the MCF7 and MDA-MB-468 cell lines (Fig. 1B) as shown by the sum of early and late apoptotic cells, with increased apoptotic rates observed in the MCF7 cells, as compared with the MDA-MD-468 cells; these results were consistent with the $\mathrm{IC}_{50}$ values of isorhamnetin that were determined for the two cell lines.

Isorhamnetin inhibited the Akt/mTOR and MEK/ERK signaling pathways, and promoted the activity of the mitochondrial apoptosis signaling pathway. In order to investigate the inhibitory mechanism underlying the effects of isorhamnetin on breast cancer cells, the PI3K/Akt/mTOR and MEK/ERK signaling pathways, which are closely associated with cell proliferation and survival, were examined. As shown in Fig. 2A, the phosphorylation levels of Akt and mTOR were markedly decreased by isorhamnetin, as well as the phosphorylation levels of MEK $1 / 2$ and ERK1/2 both in MCF7 and MDA-MB-468 cells; however, those of their upstream signaling molecules, such as phosphorylated EGFR and PI3K (110 $\alpha$ ) remained unchanged. The expression of the components of the cell apoptosis signaling pathway, including Bax, Bcl-2, Bcl-xL and cleaved caspase-3 were also investigated. Bax expression was induced by isorhamnetin, whereas the Bcl-2 expression level was markedly downregulated, and a marginal decrease was observed in the expression level of Bcl-xL (Fig. 2B). Cleaved caspase-3 expression increased, implying that cell apoptosis was induced by isorhamnetin.

As isorhamnetin exhibits anti-oxidant effects, its impact on the downstream signaling molecule, NF-кB P65 was investigated, in order to identify whether these effects contributed to its anti-tumorigenic activity. As shown in Fig. 2C,

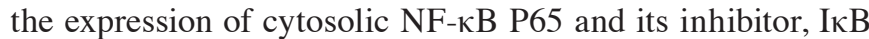
were increased by isorhamnetin, whereas nuclear NF- $\kappa$ B P65 expression was decreased, suggesting that the nuclear translo-

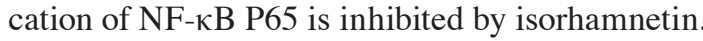

Inhibition of the Akt or MEK signaling pathways attenuates the effects of isorhamnetin. Akt and MEK inhibitors (perifosine and PD184352) were used to elucidate the mechanism of action of isorhamnetin (whether it is via the Akt or MEK signaling pathways) on breast cancer (Fig. 3). Perifosine is a potent pan-Akt inhibitor, which was used to block the Akt signaling pathway. Akt phosphorylation was inhibited by perifosine and partly inhibited by isorhamnetin (10 $\mu \mathrm{M}$; Fig. 3A, Following pretreatment of the MCF7 cells with perifosine prior to the addition of isorhamnetin, induction of cell apoptosis was only marginally increased by isorhamnetin, as determined by cleaved caspase-3 and Annexin-V/PI dual staining (Fig. 3C). These results demonstrate that blocking of Akt phosphorylation attenuated the effects of isorhamnetin.

Similarly, the MEK and ERK inhibitor, PD184352 was used to block the corresponding signaling pathways. As shown in Fig. 3B, PD184352 $(1 \mu \mathrm{M})$ and isorhamnetin $(10 \mu \mathrm{M})$ inhibited MEK1/2 phosphorylation and induced cell apoptosis. This

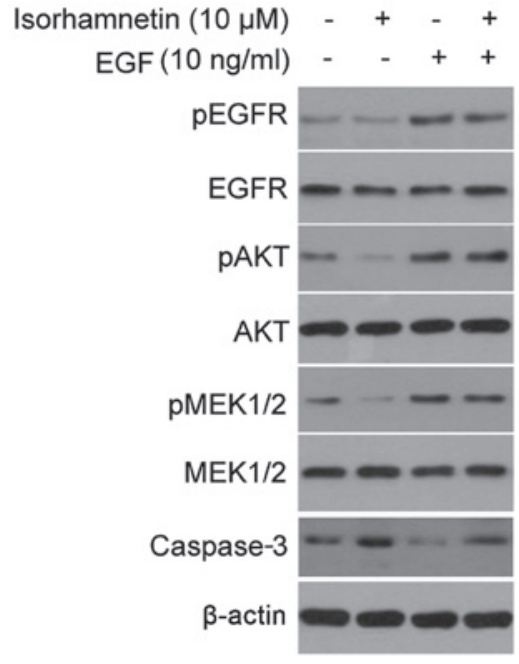

Figure 4. Apoptotic levels were decreased following treatment with EGF in the MCF7 cells. EGF induced the phosphorylation of EGFR, Akt and MEK, which reversed the inhibitory effects of isorhamnetin on Akt and MEK phosphorylation, and attenuated the levels of cell apoptosis induced by isorhamnetin, as shown by decreased levels of cleaved caspase-3. MEK, mitogen-activated protein kinase kinase; EGFR, epidermal growth factor receptor.

A

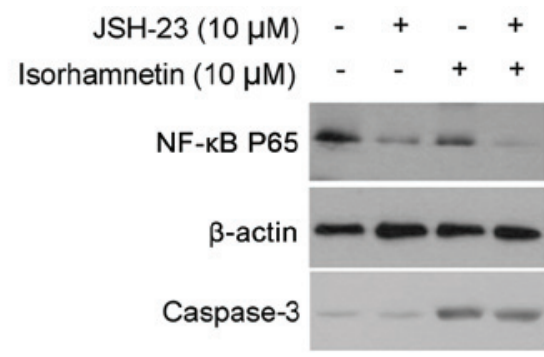

B
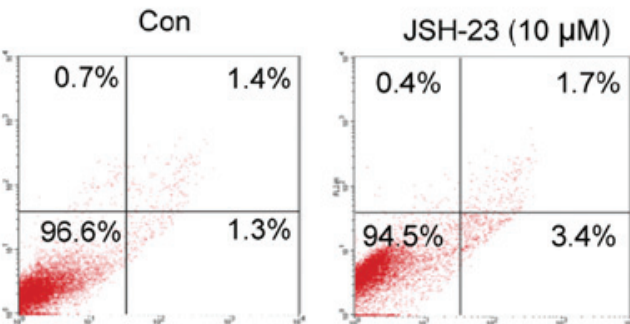

Isorhamnetin $(10 \mu \mathrm{M})$
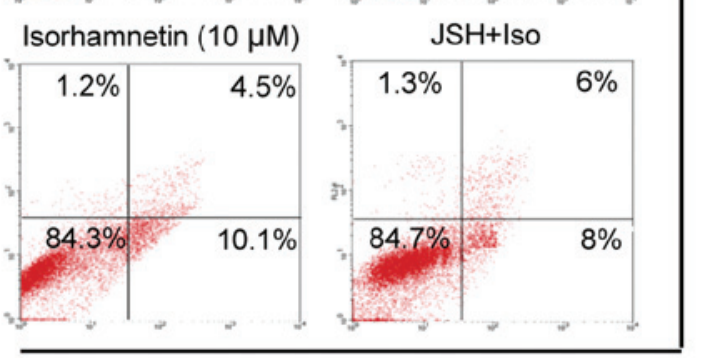

$\bar{\alpha}$

Annexin V

Figure 5. JSH-23, the NF- $\mathrm{kB}$ inhibitor, did not contribute to the induction of apoptosis by isorhamnetin in the MCF7 cells. (A) Induction of caspase-3 cleavage was not increased by treatment with JSH-23 (12-h treatment); (B) Induction of cell apoptosis was not augmented by the NF-kB inhibitor, JSH-23 (48 h treatment). NF-кB, nuclear factor- $\kappa \mathrm{B}$; Con, control; PI, propidium iodide; JSH, JSH-23; Iso, isorhamnetin.

was also determined by increased levels of cleaved caspase-3 expression and Annexin-V/PI dual staining (Fig. 3D). MCF7 
cells were pre-treated with PD184352 prior to the addition of isorhamnetin, and the induction of cell apoptosis was only marginally increased following treatment with isorhamnetin. These results demonstrate that inhibition of the MEK signaling pathway also attenuates the effects of isorhamnetin.

EGF reversed the inhibitory effects of isorhamnetin. EGF is a growth factor that binds to EGFR in order to induce the activation of downstream PI3K/Akt and MEK/ERK signaling pathways. Although EGFR phosphorylation did not change following treatment with isorhamnetin, the addition of EGF activated the Akt and MEK signaling pathways, and may be used to verify the effects of isorhamnetin. As shown in Fig. 4A, treatment with EGF markedly increased the phosphorylation levels of EGFR, Akt and MEK1/2, and these effects overrode the inhibitory effects of isorhamnetin on Akt and MEK1/2, thus inhibiting the cell apoptosis induced by isorhamnetin, as determined by decreased levels of cleaved caspase- 3 expression.

$N F-\kappa B$ does not contribute to the induction of cell apoptosis by isorhamnetin. The present study investigated whether the inhibition of NF- $\kappa \mathrm{B}$ translocation contributed to cell apoptosis. An NF- $\kappa$ B inhibitor, JSH-23, was used to block the nuclear translocation of NF- $\kappa \mathrm{B}$, prior to treatment with isorhamnetin. As shown in Fig. 5, treatment with JSH-23 inhibited NF- $\kappa$ B translocation, but did not result in the apoptosis of MCF7 cells, either alone or in combination with isorhamnetin (as demonstrated by cleaved caspase-3 and Annexin-V/PI dual staining). These results suggested that inhibition of the $N F-\kappa B$ signaling pathway does not contribute to the induction of cell apoptosis by isorhamnetin.

\section{Discussion}

The present study examined the inhibitory effects of isorhamnetin on breast cancer cell lines, and demonstrated that isorhamnetin inhibits the proliferation of various types of breast cancer cell, in ER-positive PR-positive HER2-negative cells (MCF7, T47D, BT474) and ER-PR-HER2- cells (BT-549, MDA-MB-231, MDA-MB-468), but exhibited low inhibitory activity levels against normal MCF10A breast epithelial cells, suggesting that isorhamnetin inhibited breast cancer cells independently of ER and PR, and relies instead on activated cancer signaling pathways. Isorhamnetin also induced apoptosis in breast cancer cell lines. These results indicate that isorhamnetin may act as a novel natural compound for the prevention or treatment of breast cancer.

The molecular mechanisms underlying the observed anticancer effects of isorhamnetin were also investigated. Since the Akt/mTOR and MEK/ERK signaling pathways have important roles in breast cancer, they are closely associated with endocrine therapy resistance. Therefore, the effects of isorhamnetin on these signaling pathways were analyzed, demonstrating that isorhamnetin decreased the phosphorylation levels of Akt, mTOR, MEK1 and ERK1/2, but not those of EGFR. The results indicate that isorhamnetin may act on the Akt/mTOR and MEK/ERK signaling cascades, resulting in cell apoptosis, as demonstrated by the increase in levels of Bax, Bcl-2 and cleaved caspase-3 expression. The results of the present study are concordant with those of a previous study that demonstrated that isorhamnetin induces apoptosis via the inhibition of PI3K and MEK1 $(27,30)$. A decrease in the phosphorylation levels of Akt and MEK1 was observed in the present study following pretreatment with their respective inhibitors (perifosine and PD184352). Furthermore, the inhibition of Akt and MEK was decreased by EGF, which induced activation of the PI3K/Akt/mTOR and MEK/ERK signaling pathways.

A previous study attributed the major chemopreventive mechanism of isorhamnetin to its antioxidant effects (31). Therefore, the present study investigated whether $\mathrm{NF}-\kappa \mathrm{B}$ contributed to the antitumor activity of isorhamnetin in breast cancer. The results indicate that $\mathrm{NF}-\kappa \mathrm{B}$ did not contribute to the effects of isorhamnetin on cell apoptosis and, therefore, the proposed antioxidant activity may not explain the apoptotic effects of isorhamnetin on breast cancer, which may be associated instead with other effects, such as anti-inflammatory action. The antitumor activity of isorhamnetin may be different in various cancer types, such as in colon cancer, in which isorhamnetin has been shown to inhibit c-Src activation and $\beta$-catenin nuclear translocation (32). In gastric cancer, isorhamnetin inhibits cell proliferation and invasion, and induces apoptosis through the modulation of the peroxisome proliferator-activated receptor $\gamma$ activation signaling pathway (24).

In conclusion, the results of the present study demonstrate that the antiproliferative and pro-apoptotic effects of isorhamnetin in breast cancer are mediated via inhibition of the Akt/mTOR and MEK/ERK signaling pathways, and provide a basis for pursuing the therapeutic significance and chemopreventive capabilities of isorhamnetin in breast cancer. Furthermore, isorhamnetin may be administered either alone or in combination with existing therapeutic strategies to enhance the treatment efficacy for breast cancer. However, the effects of isorhamnetin have yet to be investigated in humans, and hence further studies in humans are required prior to its clinical application to breast cancer treatment.

\section{Acknowledgements}

The present study was supported by a grant from the Science and Technology Bureau of Shaoxing (grant no. 2014B70079).

\section{References}

1. American Cancer Society. Cancer Facts \& Figures 2013. American Cancer Society, Atlanta, 2013.

2. Chen W, Zheng R, Zhang S, Zhao P, Li G, Wu L and He J: Report of incidence and mortality in China cancer registries, 2009. Chin J Cancer Res 25: 10-21, 2013.

3. Ferlay J, Steliarova-Foucher E, Lortet-Tieulent J, Rosso S, Coebergh JW, Comber H, Forman D and Bray F: Cancer incidence and mortality patterns in Europe: Estimates for 40 countries in 2012. Eur J Cancer 49: 1374-1403, 2013.

4. Holohan C, Van Schaeybroeck S, Longley DB and Johnston PG: Cancer drug resistance: An evolving paradigm. Nat Rev Cancer 13: 714-726, 2013.

5. Massarweh S and Schiff R: Resistance to endocrine therapy in breast cancer: Exploiting estrogen receptor/growth factor signaling crosstalk. Endocr Relat Cancer 13 (Suppl 1): S15-S24, 2006.

6. Geyer CE, Forster J, Lindquist D, Chan S, Romieu CG, Pienkowski T, Jagiello-Gruszfeld A, Crown J, Chan A, Kaufman B, et al: Lapatinib plus capecitabine for HER2-positive advanced breast cancer. N Engl J Med 355: 2733-2743, 2006. 
7. Guarneri V and Conte P: Metastatic breast cancer: Therapeutic options according to molecular subtypes and prior adjuvant therapy. Oncologist 14: 645-656, 2009.

8. Fedele P, Calvani N, Marino A, Orlando L, Schiavone P, Quaranta A and Cinieri S: Targeted agents to reverse resistance to endocrine therapy in metastatic breast cancer: Where are we now and where are we going? Crit Rev Oncol Hematol 84: 243-251, 2012.

9. Miller TW, Balko JM and Arteaga CL: Phosphatidylinositol 3 -kinase and antiestrogen resistance in breast cancer. J Clin Oncol 29: 4452-4461, 2011.

10. Pérez-Tenorio G and Stål O; Southeast Sweden Breast Cancer Group: Activation of Akt/PKB in breast cancer predicts a worse outcome among endocrine treated patients. Br J Cancer 86 $540-545,2002$

11. deGraffenried LA, Friedrichs WE, Russell DH, Donzis EJ, Middleton AK, Silva JM, Roth RA and Hidalgo M: Inhibition of mTOR activity restores tamoxifen response in breast cancer cells with aberrant Akt Activity. Clin Cancer Res 10: 8059-8067, 2004.

12. Kirkegaard T, Witton CJ, McGlynn LM, Tovey SM, Dunne B, Lyon A and Bartlett JM: Akt activation predicts outcome in breast cancer patients treated with tamoxifen. J Pathol 207: 139-146, 2005.

13. Tokunaga E, Kimura Y, Mashino K, Oki E, Kataoka A, Ohno S, Morita M, Kakeji Y, Baba $\mathrm{H}$ and Maehara Y: Activation of $\mathrm{PI} 3 \mathrm{~K} / \mathrm{Akt}$ signaling and hormone resistance in breast cancer. Breast Cancer 13: 137-144, 2006.

14. Donovan JC, Milic A and Slingerland JM: Constitutive MEK/MAPK activation leads to p27(Kipl) deregulation and antiestrogen resistance in human breast cancer cells. J Biol Chem 276: 40888-40895, 2001.

15. Jin W, Wu L, Liang K, Liu B, Lu Y and Fan Z: Roles of the PI-3K and MEK pathways in Ras-mediated chemoresistance in breast cancer cells. Br J Cancer 89: 185-191, 2003.

16. Normanno N, De Luca A, Maiello MR, Campiglio M,Napolitano M, Mancino M, Carotenuto A, Viglietto G and Menard S: The MEK/MAPK pathway is involved in the resistance of breast cancer cells to the EGFR tyrosine kinase inhibitor gefitinib. J Cell Physiol 207: 420-427, 2006.

17. Yao H, Xu W, Shi X and Zhang Z: Dietary flavonoids as cancer prevention agents. J Environ Sci Health C Environ Carcinog Ecotoxicol Rev 29: 1-31, 2011.

18. Asensi M, Ortega A, Mena S, Feddi F and Estrela JM: Natural polyphenols in cancer therapy. Crit Rev Clin Lab Sci 48: 197-216, 2011.

19. Cushnie TP and Lamb AJ: Recent advances in understanding the antibacterial properties of flavonoids. Int J Antimicrob Agents 38: 99-107, 2011

20. Gupta SC, Kim JH, Prasad S and Aggarwal BB: Regulation of survival, proliferation, invasion, angiogenesis and metastasis of tumor cells through modulation of inflammatory pathways by nutraceuticals. Cancer Metastasis Rev 29: 405-434, 2010.
21. Ma G, Yang C, Qu Y, Wei H, Zhang T and Zhang N: The flavonoid component isorhamnetin in vitro inhibits proliferation and induces apoptosis in Eca-109 cells. Chem Biol Interact 167: 153-160, 2007.

22. Suomela JP, Ahotupa M, Yang B, Vasankari T and Kallio H: Absorption of flavonols derived from sea buckthorn (Hippophaë rhamnoides L.) and their effect on emerging risk factors for cardiovascular disease in humans. J Agric Food Chem 54: 7364-7369, 2006.

23. Shi C, Fan LY, Cai Z, Liu YY and Yang CL: Cellular stress response in Eca-109 cells inhibits apoptosis during early exposure to isorhamnetin. Neoplasma 59: 361-369, 2012.

24. Ramachandran L, Manu KA, Shanmugam MK, Li F, Siveen KS, Vali S, Kapoor S, Abbasi T, Surana R, Smoot DT, et al: Isorhamnetin inhibits proliferation and invasion and induces apoptosis through the modulation of peroxisome proliferator-activated receptor $\gamma$ activation pathway in gastric cancer. J Biol Chem 287: 38028-38040, 2012.

25. Boubaker J, Ben Sghaier M, Skandrani I, Ghedira K and Chekir-Ghedira L: Isorhamnetin 3-O-robinobioside from Nitraria retusa leaves enhance antioxidant and antigenotoxic activity in human chronic myelogenous leukemia cell line K562. BMC Complement Altern Med 12: 135, 2012

26. Boubaker J, Bhouri W, Ben Sghaier M, Ghedira K, Dijoux Franca MG and Chekir-Ghedira L: Ethyl acetate extract and its major constituent, isorhamnetin 3-O-rutinoside, from Nitraria retusa leaves, promote apoptosis of human myelogenous erythroleukaemia cells. Cell Prolif 44: 453-461, 2011.

27. Kim JE, Lee DE, Lee KW, Son JE, Seo SK, Li J, Jung SK, Heo YS, Mottamal M, Bode AM, et al: Isorhamnetin suppresses skin cancer through direct inhibition of MEK1 and PI3-K. Cancer Prev Res (Phila) 4: 582-591, 2011.

28. Jaramillo S, Lopez S, Varela LM, Rodriguez-Arcos R, Jimenez A, Abia R, Guillen R and Muriana FJ: The flavonol isorhamnetin exhibits cytotoxic effects on human colon cancer cells. J Agric Food Chem 58: 10869-10875, 2010.

29. Lee HJ, Lee HJ, Lee EO, Ko SG, Bae HS, Kim CH, Ahn KS, Lu J and Kim SH: Mitochondria-cytochrome C-caspase-9 cascade mediates isorhamnetin-induced apoptosis. Cancer Lett 270: 342-353, 2008.

30. Li C, Yang X, Chen C, Cai S and Hu J: Isorhamnetin suppresses colon cancer cell growth through the PI3K-Akt-m TOR pathway. Mol Med Rep 9: 935-940, 2014.

31. Di Carlo G, Mascolo N, Izzo AA and Capasso F: Flavonoids: Old and new aspects of a class of natural therapeutic drugs. Life Sci 65: 337-353, 1999.

32. Saud SM, Young MR, Jones-Hall YL, Ileva L, Evbuomwan MO, Wise J, Colburn NH, Kim YS and Bobe G: Chemopreventive activity of plant flavonoid isorhamnetin in colorectal cancer is mediated by oncogenic Src and $\beta$-catenin. Cancer Res 73: 5473-5484, 2013. 